\title{
Surgery for Diabetes
}

\author{
Ricardo Cohen • Pedro Paulo Caravatto • \\ Tarissa Petry
}

Published online: 15 May 2013

(c) Springer Science + Business Media New York 2013

\begin{abstract}
Bariatric surgery was initially developed as a tool for weight reduction only, but it is gaining increasing popularity because of its remarkable effect on glucose metabolism in morbidly obese and less obese patients. Recent publications have shown the superiority of metabolic surgery over medical treatment for diabetes, creating a new field of clinical research that is currently overflowing in the medical community with outstanding high-quality data. Metabolic surgery is effective in treating diabetes, even in non-morbidly obese patients.
\end{abstract}

Keywords Type 2 diabetes mellitus - Diabetes .

Metabolic surgery · Bariatric surgery · Obesity ·

Gastrointestinal surgery · Glycemic control

\section{Introduction}

During the past decades, the prevalence of type 2 diabetes (T2DM) in the United States has more than doubled, indicating an epidemic. Approximately 25.8 million Americans were diagnosed with diabetes (nearly $8.3 \%$ of the population) in 2010, and by 2050, as many as one in three US adults could have diabetes $[1 \bullet, 2]$. Furthermore, T2DM is the leading cause of kidney failure, nontraumatic lower-limb amputations, coronary heart disease, stroke, and visual impairment among adults in the United States [3].

Aside from high morbidity and premature mortality, T2DM imposes a substantial burden on the economy

R. Cohen $(\bowtie) \cdot$ P. P. Caravatto · T. Petry

The Center of Excellence of Metabolic and Bariatric Surgery,

Hospital Oswaldo Cruz, Rua Padre João Manuel, 222 ste 130,

Sao Paulo, SP 01411-000, Brazil

e-mail: ricardo.cohen@haoc.com.br because of its increasing medical costs and indirect costs, which are the result of work absenteeism, reduced productivity, and unemployment from chronic disability. In 2012, the American Diabetes Association (ADA) estimated that the total national cost of diabetes in the United States was $\$ 245$ billion, of which more than $70 \%$ represent direct health expenditures [1•]. This represents a $41 \%$ increase compared with a previous estimate published in 2008 [4]. There is also a significant increase in the use of health resources by people with diabetes. For example, diabetics account for $\sim 25 \%$ of all US hospital inpatient days, almost one-third of all nursing/resident facilities days, and approximately half of all physician offices, emergency departments, and hospital outpatient visits. An even worse scenario has been reported when analyzing data by age, in particular, the elderly $[1 \bullet]$.

Several clinical trials [including the Diabetes Control and Complications Trial (DCCT) and the United Kingdom Prospective Diabetes Study (UKPDS)] have established that glycemic control is a major step to prevent microvascular complications. However, to prevent disease progression and cardiovascular disease, a broader management, focusing on lipids, blood pressure and glycemia, is necessary $[5,6]$. Currently, the ADA recommends that most adults with T2DM achieve a glycated hemoglobin (A1c) below $7 \%$, blood pressure below $130 \times 80 \mathrm{mmHg}$, and low-density lipoprotein (LDL) cholesterol levels below $100 \mathrm{mg} / \mathrm{dL}$ [7].

For that purpose, new antidiabetic medications, such as glucagon-like peptide-1 (GLP-1) analogs and dipeptidyl peptidase-4 (DPP-4) inhibitors, have been developed. Nonetheless, diabetes control, which has improved in the last decade, remains elusive, despite the different treatment combinations that are now available. Less than $20 \%$ of the North American population is able to achieve metabolic control (Table 1) [8,9]. 
Table 1 Prevalence of achieving A1c, BP, LDL cholesterol goal levels according to the ADA

\begin{tabular}{lll}
\hline & $1988-1994(\%)$ & $2007-2010(\%)$ \\
\hline A1c $<7 \%$ & 43 & 52.5 \\
BP $<130 \times 80 \mathrm{mmHg}$ & 33.2 & 51.1 \\
LDL cholesterol $<100 \mathrm{mg} / \mathrm{dL}$ & 9.9 & 56.2 \\
All three combined & 1.7 & 18.8 \\
\hline
\end{tabular}

Alc glycated hemoglobin, $B P$ blood pressure, $L D L$ low-density lipoprotein

Prospective longitudinal studies have shown that bariatric surgery is the most effective intervention for obesity and its associated diseases, in particular T2DM. Bariatric surgery reduces the long-term mortality rate of morbidly obese patients compared with medical treatment $[10,11]$.

Improvement of metabolic control in the post-operative population was observed days or weeks after the surgical procedure, long before considerable weight loss, precluding a direct antidiabetic effect. Because of this finding, some procedures began to be considered as an option to treat less obese patients who have uncontrolled T2DM [12].

The medical community is still skeptical when accepting surgery as a treatment modality for T2DM, particularly in less obese individuals, fearing a theoretical risk of nutritional complications. However, the full risk-benefit ratio of surgical versus medical approaches to treat T2DM has not been adequately ascertained until recently.

During 2012, data supporting surgery for diabetes has greatly increased. Two randomized controlled trials (RCTs) comparing bariatric surgery with medical therapy for T2DM were published, and both reported better glycemic control, T2DM remission, and reduction of cardiovascular risk factors

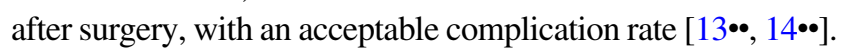

Despite these compelling results, additional hard data are required so that gastrointestinal surgery can be validated as an alternative therapy in uncontrolled diabetic patients with a lower body mass index (BMI). From a surgeon's point of view, we are on the right track as emerging clinical evidence is being reported on good metabolic outcomes regardless of patient's BMI.

\section{Metabolic Surgery: Is the Name Important?}

Initial reports often used the term diabetes surgery to define any gastrointestinal surgery that intended to treat T2DM. At that time, it appeared reasonable to differentiate diabetes surgery from bariatric surgery because their primary objectives were different (weight loss vs. glucose control), although surgical procedures were initially the same [15].

A more recent concept emerged and the term metabolic surgery was adopted by field experts. This indicated that gastrointestinal surgery not only promotes good glucose control, but also promotes the control of metabolic illnesses, including hypertension and dyslipidemia [16].

There is also a misconception that metabolic surgery is an investigational approach to nonobese diabetic patients and comprises experimental procedures that are limited to a few case series. Metabolic surgery is more involved than this definition. This is a new discipline in the field of gastrointestinal surgery, shifting its primary focus and indication criteria, allowing new types of procedures to be used and changing the way that outcomes are evaluated (Table 2) [17•]. More importantly, in pursuing mechanisms of actions related to metabolic control, metabolic surgery challenges the most skilled surgeon to go back to basic science. Pories's et al. [18] landmark paper about diabetes surgery suggested that an operation not only proves to be the most effect therapy for adult-onset diabetes mellitus, but also changes the way bariatric surgeons think.

\section{Effects of Bariatric Surgery on Glycemic Control}

Numerous data obtained from observational, nonrandomized, and randomized trials have demonstrated the safety and efficacy of gastrointestinal operations, mainly Rouxen-Y gastric bypass (RYGB), sleeve gastrectomy (SG), biliopancreatic diversion (BPD), and adjustable gastric banding in treating T2DM for morbidly obese and less obese patients $[10,11,13 \bullet \bullet, 14 \bullet \bullet, 18,19,20 \bullet \bullet, 21]$. Although glycemic control following adjustable gastric banding results solely from significant weight loss, other procedures promote glycemic control within weeks or even days following surgery [22]. This remarkable outcome results from mechanisms beyond reduced food intake and weight loss, precluding a direct antidiabetic effect.

Based on these results and others observed following bariatric surgery in terms of metabolic control in morbidly obese patients, several studies were conducted aiming to treat patients with a BMI below $35 \mathrm{~kg} / \mathrm{m}^{2}$ and uncontrolled metabolic diseases. As expected, good results regarding metabolic control were obtained without significant weight loss, particularly after RYGB $[19,20 \bullet \bullet$.

We recently published (20) the largest and longest-term study to date to examine the safety and efficacy of RYGB in T2DM patients with a BMI between 30 and $35 \mathrm{~kg} / \mathrm{m}^{2}$. All patients were followed from 1 to 6 years (median 5 years). Main outcomes were safety and T2DM remission, defined as A1c $<6.5 \%$ without medication. All patients had uncontrolled T2DM, despite appropriate lifestyle modifications and use of antidiabetic medications and/or insulin for at least 1 year. The mean preoperative A1c was $9.7 \%$ and the mean duration of disease was 12.5 years. T2DM remission was observed in $88 \%$ of patients, with a significant reduction in A1c (9.7-5.9 \%), despite cessation of diabetes medication in 
Table 2 Bariatric versus metabolic surgery

\begin{tabular}{lll}
\hline & Bariatric & Metabolic \\
\hline $\begin{array}{l}\text { Purpose } \\
\text { Indication criteria }\end{array}$ & Weight loss & $\begin{array}{l}\text { Glycemic/metabolic control + CV risk reduction } \\
\text { Uncontrolled T2DM, WC, insulin resistance surrogate } \\
\text { markers, NASH, uncontrolled metabolic parameters, } \\
\text { increased CV risks }\end{array}$ \\
$\begin{array}{l}\text { Type of procedures } \\
\text { Measures of outcomes }\end{array}$ & "Traditional”: RYGB, SG, BPD, AGB & $\begin{array}{l}\text { Traditional + DJB, IT, Endoscopic duodenal-jejunal liner } \\
\text { Presumed mechanisms of action }\end{array}$ \\
\hline
\end{tabular}

Adapted from Rubino and Cummings [17]

$C V$ cardiovascular, $B M I$ body mass index, $T 2 D M$ type 2 diabetes mellitus, $W C$ waist circumference, $N A S H$ non-alcoholic steatohepatitis, $R Y G B$ Roux-en-Y gastric bypass, $S G$ sleeve gastrectomy, $A G B$ adjustable gastric banding, $D J B$ duodenal-jejunal bypass, $I T$ ileal transposition

the majority of cases. There was significant lipid control (LDL cholesterol and triglycerides). After inserting information in the UKPDS cardiovascular risk engine (http:// www.dtu.ox.ac.uk/riskengine/), a significant 10-year decrease in fatal and nonfatal stroke and myocardial infarction was found. No correlation was found between metabolic outcomes and weight loss. Beta cell sensitivity significantly improved as measured by C-peptide response to glucose load. No mortality, major surgical complication, or massive weight loss was reported [20••].

Our research group searched for an alternative type of procedure that could lead to none or mild weight loss and still achieve metabolic control in a population that, in theory, does not need massive weight loss. We pioneered duodenal-jejunal bypass (DJB) in clinical research. This procedure prevents direct contact between nutrients and the duodenum and proximal jejunum without any gastric restriction or exclusion [23].

We performed a longitudinal prospective study of 36 diabetic patients with a mean BMI of $28.4 \mathrm{~kg} / \mathrm{m}^{2}$ who were submitted to DJB and full remission (A1c $<7 \%$; fasting plasma glucose $[\mathrm{FPG}]<126 \mathrm{mg} / \mathrm{dL}$ ) was achieved by $40 \%$ of patients at 1-year of follow-up [24].

Finally, a new tool for T2DM control was introduced. The DJB liner (DJBL) is an endoscopically-placed device, which prevents contact between partially digested nutrients and the proximal intestine. The DJBL mimics exclusion of the proximal bowel, which is a component of several effective metabolic surgeries [25]. Escalona et al. [26•] implanted the DJBL in 39 morbidly obese patients and found that all patients achieved good loss of excess body weight $(47.0 \pm 4.4 \%)$ after 12 months. These patients also showed a significant improvement in waist circumference, blood pressure, total and LDL cholesterol levels, triglyceride levels, and FPG levels.

Cohen et al. [27•] recently published the only report in the literature regarding low-BMI T2DM. Sixteen of 20 subjects implanted with the DJBL completed the 1-year study (mean BMI of $\left.30 \mathrm{~kg} / \mathrm{m}^{2}\right)$. Ten of 16 subjects $(62.5 \%)$ who completed the study demonstrated A1c $<7 \%$ at week 52, and statistically significant lipid control was achieved (LDL and triglycerides). No significant correlations between changes in body weight and changes in FPG or A1c were observed. Based on the results of that study, the DJBL appears to reproduce some aspects of metabolic surgery in terms of its ability to improve A1c, FPG, and lipid parameters without a direct relation to weight modification. Interestingly, after some mathematical modeling of data extracted from an oral test after a mixed meal challenge glucose excursion, C-peptide deconvolution, and insulin curves, we found almost immediate, weight loss-independent improved insulin sensitivity after placement of the DJBL (unpublished data). Moreover, this effect was maintained throughout the year when the device was kept in place. No improvement in insulin secretion was observed. The DJBL appears to be an effective tool for metabolic control, allowing some potential associations with GLP-1 analogs and DPP-4 inhibitors because the device itself might not change insulin secretion. The DJBL is an outpatient-based endoscopic procedure that is associated with virtually no major complications. However, the main drawback of the DJBL is that its design is recommended for only 1 year of implantation. It also is pending FDA approval within the United States. The DJBL may be a good screening tool for the effectiveness of duodenal exclusion before a surgical procedure, or an excellent way to quickly improve glycemic and metabolic control in individuals with glucotoxicity and lipotoxicity who may need prompt, effective, and safe intervention.

Well-designed RCTs are required to provide additional data showing that metabolic surgery may lead to better outcomes compared with standard clinical treatment. They are also required to test the efficacy and safety of metabolic surgery in treating low BMI diabetic patients. RCTs were performed by Schauer et al. [13••], who studied RYGB and SG, and by Mingrone et al. [14••], who analyzed RYGB and BPD, all of which were compared with standard medical therapy and lifestyle modifications (Table 3). 
Table 3 Randomized controlled trials of metabolic surgery from 2012

\begin{tabular}{|c|c|c|}
\hline & Mingrone et al. $[14 \bullet \bullet]$ & Schauer et al. $\left[13^{\bullet \bullet}\right]$ \\
\hline Interventions & $\mathrm{RYGB} \times \mathrm{BPD} \times$ meds & $\mathrm{RYGB} \times \mathrm{SG} \times$ meds \\
\hline Follow-up & 2 years & 1 years \\
\hline Number of patients & 60 & 150 \\
\hline $\mathrm{BMI}\left(\mathrm{kg} / \mathrm{m}^{2}\right)$ & $>35$ & $27-43$ \\
\hline T2DM duration (years) & 6 & $>8$ \\
\hline Baseline A1c (\%) & 8.7 & 9.2 \\
\hline Definition of remission & FPG $<100 \mathrm{mg} / \mathrm{dL}$ and A1c $<6.5 \%$ without meds & A1c $<6 \%$ with or without meds \\
\hline $\mathrm{T} 2 \mathrm{DM}$ remission & $75 \%$ RYGB, $95 \%$ BPD, $0 \%$ meds & $42 \%$ RYGB, $37 \%$ SG, $12 \%$ meds \\
\hline
\end{tabular}

$R Y G B$ Roux-en-Y gastric bypass, BPD biliopancreatic diversion, $S G$ sleeve gastrectomy, $B M I$ body mass index, $T 2 D M$ type 2 diabetes mellitus, $A 1 c$ glycated hemoglobin, $F P G$ fasting plasma glucose

In a study not powered for lower BMIs, without specific details, Schauer et al. [13••] showed better glycemic control (and better weight loss) in the surgical arms than the medical arm. In their study, which included morbidly obese patients, RYGB had similar results over glycemic control as $\mathrm{SG}$.

More recently, Kashyap et al. [28•] published an extended follow-up (24 months) of the previous study. They found more durable glycemic control in the RYGB group, with a substantially greater number of patients to achieve an A1c $<6 \%$, despite similar weight loss compared with SG. Lastly, RYGB had a greater effect on beta cell function and reduction of truncal fat compared with SG and medical management (Table 4).

Mingrone et al. [14・•] also reported improved glycemic control in morbidly obese T2DM patients, as well as a reduction in cardiovascular risk factors, including dyslipidemia and hypertension, in the surgical arms compared with medical treatment. T2DM remission rates in the surgical group were dramatic, especially in the BPD group. Additionally, no correlation was observed between preoperative BMI, weight loss, and glucose control, which occurred within 15 days after surgery, preceding substantial weight loss.

Table 4 Extended (24 months) follow-up of metabolic surgery

\begin{tabular}{llll}
\hline & RYGB & SG & Medical \\
\hline A1c $(\%)$ & 6.7 & 7.1 & 8.4 \\
BMI $\left(\mathrm{kg} / \mathrm{m}^{2}\right)$ & 27.4 & 28.2 & 35.6 \\
Insulin sensitivity & Increase by 2.7 times & Unchanged & Unchanged \\
$\begin{array}{l}\text { Beta-cell function } \\
\text { Increase by 5.8 times }\end{array}$ & Unchanged & Unchanged \\
$\begin{array}{c}\text { Truncal fat } \\
\text { change from }\end{array}$ & -15.9 & -10.1 & +0.9 \\
baseline $(\%)$ & & & \\
\hline
\end{tabular}

Data from Kashyap et al. [28•]

$R Y G B$ Roux-en-Y gastric bypass, $S G$ sleeve gastrectomy, $A 1 c$ glycated hemoglobin, $B M I$ body mass index

\section{Mechanisms}

Several advances in understanding mechanistic aspects of metabolic surgery have been reported, supporting weight loss independent and even incretin independent antidiabetic effects of different operations. These advances are challenging the former pure foregut and hindgut theories and the major role of incretins over postoperative glycemic control $\left[29,30^{\bullet}\right]$.

Breen et al. [31•] performed DJB in lean streptozotocininduced uncontrolled diabetes rats. Their results suggested that activation of jejunal nutrient-sensing mechanisms mediates the ability of DJB to rapidly lower glucose concentrations in insulin-deficient, uncontrolled diabetes, independently of weight loss. DJB normalized glucose concentrations independently of changing insulin levels, food intake, and body weight.

Jiao et al. [32•] performed DJB on obese, insulin-resistant Zucker fa/fa rats, and showed restoration of insulin sensitivity in liver and peripheral tissues independent of body weight. Restoration of normoglycemia was attributable to enhancement in key insulin-signaling molecules, including insulin receptor substrate-2, and substrate metabolism. This was achieved through a multifaceted mechanism involving activation of AMP-activated protein kinase and downregulation of key regulatory genes involved in lipid and glucose metabolism. In the study by Jiao et al., rapid normalization of hepatic gluconeogenic capacity and basal hepatic glucose production required intact vagal innervation. DJB selectively altered the tissue concentrations of intestinally derived glucoregulatory hormone peptides in a segment-specific manner. These data support the significance of vagal input and intestinal hormone peptides toward normalization of glucose and lipid homeostasis after DJB.

Salinari et al. [33•] tested the effects of proteins from duodenum to jejunum conditioned medium of $\mathrm{db} / \mathrm{db}$ or Swiss mice on glucose uptake in vivo in Swiss mice and in vitro in Swiss mice (insulin sensitive) soleus and L6 
cells. They studied the effect of sera and conditioned medium proteins from insulin resistant and insulin-sensitive subjects on insulin signaling in human myoblasts. They found that jejunal proteins from $\mathrm{db} / \mathrm{db}$ mice or from insulin resistant subjects impaired muscle insulin signaling, thus inducing insulin resistance. This study demonstrated that the small intestine of insulin-resistant humans and mice secretes a protein factor(s) inducing insulin resistance by impairing insulin signaling. This is a major advance towards the identification of a possible duodenal factor that may worsen insulin resistance in T2DM patients. Another important finding in that study is the lack of major importance of the role of GLP-1 after SG to promote improvement in glucose homeostasis and weight loss. GLP-1 secretion is greatly enhanced in rats and humans following some bariatric procedures, including SG. Wilson-Perez et al. [34] tested this hypothesis using two separate models of GLP-1 receptor deficiency. They found that SG-operated GLP-1 receptor-deficient mice responded similarly to wild-type controls in terms of body weight and body fat loss, improved glucose tolerance, food intake reduction, and altered food selection. These data demonstrate that GLP-1 receptor activity is not necessary for metabolic improvement induced by SG surgery, indicating that other mechanisms are involved in metabolic improvement after SG.

Another important mechanism that might help understand the weight loss and metabolic improvement after RYGB was investigated by Liou et al. [35•]. They used a mouse model of gastric bypass surgery to characterize changes in the gut microbiota, both temporally and along the length of the gastrointestinal tract. Gastric bypass induced substantial, rapid, and sustained changes to the gut microbial communities. These changes were independent of diet and weight loss associated with this procedure. Transfer of the surgically altered microbial community to nonoperated, germ-free mice resulted in weight loss and decreased body fat. These observations show that specific alterations in the gut microbiota contribute to the beneficial effects of bariatric surgery on energy balance and obesity, and eventually metabolic control.

\section{Balancing the Role of Weight Loss in T2DM Control and Cardiovascular Events}

Lifestyle interventions to prevent T2DM have not avoided cardiovascular events, even with long-term follow-up [36, 37]. Sjöström et al. [38••], in the Swedish Obese Subjects study, a nonrandomized prospective study, concluded that bariatric surgery led to a reduced number of cardiovascular events and deaths compared with usual care. One of the most important findings in that study was the lack of a relation between BMI and cardiovascular mortality and/or events, while there was a strong correlation between fasting insulin levels and cardiovascular events/deaths. The Look AHEAD trial [39] has already shown that intensive lifestyle modification can induce significant improvements in other intermediate health indicators, such as body weight, fitness, blood pressure, glycemic control, and lipids. However, these improved risk factor profiles did not translate into lower rates of nonfatal myocardial infarction, nonfatal stroke, hospitalization for angina, or death compared with conventional diabetes treatment [40]. The Look AHEAD trial found that people who are obese and have type 2 diabetes can lose weight and maintain their weight loss with a lifestyle intervention. Although this study found that weight loss had many positive health benefits for people with T2DM, it did not reduce the number of cardiovascular events (this was the primary endpoint of that study), and therefore, it was halted (National Institutes of Health News, Oct 19, 2012). There is increasing evidence that metabolic surgery is more effective than medical or lifestyle interventions for weight loss and diabetes remission. In a recent analysis by Arterburn et al. [41 •] of severely obese patients with diabetes, the adjusted probability of initial remission was 12- to 24-fold greater for the 1,395 patients who had bariatric surgery than for the 62,322 who received usual medical care alone.

In a recent Editorial, the same author [42•] stated that the improvement in T2DM after intensive lifestyle treatment was more prominent in patients with early stage diabetes. This is similar to what has been reported for bariatric surgery, where better results are linked to a milder, earlier stage disease. Based on these findings, a strong argument can be made for considering earlier surgical intervention in moderately obese patients (BMI $<30 \mathrm{~kg} / \mathrm{m}^{2}$ ) with early stage diabetes, mainly in those with severe insulin resistance, a higher cardiovascular risk, and uncontrolled disease. This supports the 2011 International Diabetes Federation statement on new perspectives on surgical treatment of uncontrolled T2DM in patients with BMIs between 30 and $35 \mathrm{~kg} / \mathrm{m}^{2}$ [43••].

Timing is of the essence for T2DM remission. In a retrospective study, Arterburn again found that almost $35 \%$ of patients who went into remission following RYGB redeveloped T2DM within 5 years of surgery. Predictive factors for remission were poor preoperative glycemic control, insulin use, and longer diabetes duration. This suggests that the earlier the intervention takes place, the better the outcome [41•].

Look AHEAD fundamentally supports the idea that weight loss should not be considered as the means to reduce T2DM-associated cardiovascular disease, but rather as an added benefit to interventions aimed at improving diabetes (diet, surgery, etc.). Instead, weight loss should be considered as a means for achieving good metabolic [44]. 


\section{Conclusions}

As was first proposed by the International Diabetes Federation in 2011, recent data now show the superiority of metabolic surgery compared with medical treatment for diabetes control. We believe that we are on the right track towards validating metabolic surgery as an option for uncontrolled, high cardiovascular risk, diabetic patients. However, some questions remain unanswered [44], and new RCTs should attempt to answer the following questions. (1) How soon can we offer metabolic surgery in T2DM evolution? (2) Should we expand indications for metabolic surgery to more than BMI? (3) If so, what should be the additional parameters be? (4) Can we provide additional data regarding the benefits of surgery over medical treatment, such as microvascular disease resolution? (5) What are the mechanisms involved in glucose control? Will we be able to test them in clinical studies?

By answering those questions we should add metabolic surgery to future guidelines for T2DM treatment.

Disclosures Ricardo Cohen has served on a board for GI Dynamics and has received grant support from Covidien, Johnson Medical Brasil, and GI Dynamics. Pedro Paulo Caravatto and Tarissa Petry declare that they have no conflict of interest.

Compliance with Ethics Guidelines This article does not contain any studies with human or animal subjects performed by any of the authors.

\section{References}

Papers of particular interest, published recently, have been highlighted as:

- Of importance

•- Of major importance

1. - American Diabetes Association. Economic costs of diabetes in the U.S. in 2012. Diabetes Care 2013;36:1033-46. An update on the soaring costs of treating type 2 diabetes.

2. Boyle JP, Thompson TJ, Gregg EW, Barker LE, Williamson DF. Projection of the year 2050 burden of diabetes in the US adult population: dynamic modeling of incidence, mortality, and prediabetes prevalence. Popul Health Metr. 2010;8:29.

3. Lebovitz HE. Science, clinical outcomes and the popularization of diabetes surgery. Curr Opin Endocrinol Diabetes Obes. 2012;19:359-66.

4. American Diabetes Association. Economic costs of diabetes in the U.S. in 2007. Diabetes Care. 2008;31:596-615.

5. Murray P, Chune GW, Raghavan VA. Legacy effects from DCCT and UKPDS: what they mean and implications for future diabetes trials. Curr Atheroscler Rep. 2010;12:432-9.

6. Gaede P, Lund-Andersen H, Parving H-H, Pedersen O. Effect of a multifactorial intervention on mortality in type 2 diabetes. N Engl J Med. 2008;358:580-91.
7. American Diabetes Association. Standards of medical care in diabetes 2011. Diabetes Care. 2011;34:S11-61.

8. Stark Casagrande S, Fradkin JE, Saydah SH, Rust KF, Cowie CC. The prevalence of meeting A1C, blood pressure, and LDL goals among people with diabetes, 1988-2010. Diabetes Care 2013. doi: $10.2337 / \mathrm{dc} 12-2258$.

9. Grant RW, Buse JB, Meigs JB, University Health System Consortium (UHC) Diabetes Benchmarking Project Team. Quality of diabetes care in U.S. academic medical centers: low rates of medical regimen change. Diabetes Care. 2005;28:337-442.

10. Sjöström L, Lindroos A-K, Peltonen M, et al. Lifestyle, diabetes and cardiovascular risk factors 10 years after bariatric surgery. N Engl J Med. 2004;351:2683-93.

11. Sjöström L, Narbro K, Sjöström CD, et al. Effects of bariatric surgery on mortality in Swedish obese subjects. N Engl J Med. 2007;357:741-52.

12. Rubino F. Is type 2 diabetes an operable intestinal disease? A provocative yet reasonable hypothesis. Diabetes Care. 2008;31:S290-6.

13. $\bullet$ Schauer PR, Kashyap SR, Wolski K et al. Bariatric surgery vs. intensive medical therapy in obese patients with diabetes. N Engl J Med. 2012;366:1567-76.

14. • Mingrone G, Panunzi S, De Gaetano A et al. Bariatric surgery versus conventional medical therapy for type 2 diabetes. N Engl J Med. 2012;366:1577-85. First 2 randomized controlled trials comparing gastrointestinal surgery versus medical treatment focusing metabolic outcomes.

15. Rubino F, Kaplan LM, Schauer PR, Cummings DE, Diabetes Surgery Summit Delegates. The Diabetes Surgery Summit consensus conference: recommendations for the evaluation and use of gastrointestinal surgery to treat type 2 diabetes mellitus. Ann Surg. 2010;251:399-405.

16. Rubino F, Shukla A, Pomp A et al. Bariatric, metabolic, and diabetes surgery: what's in a name? Ann Surg. 2013. doi:10.1097/ SLA.0b013e3182759656.

17. - Rubino F, Cummings DE. Surgery: the coming age of metabolic surgery. Nat Rev Endocrinol. 2012;8:702-4. Interesting review of the foundation of a new discipline-Metabolic Surgery- and its implications in the care of diabetes and metabolic syndrome.

18. Pories WJ, Swanson MS, MacDonald KG, et al. Who would have thought it? An operation proves to be the most effective therapy for adult-onset diabetes mellitus. Ann Surg. 1995;222:339-50.

19. Schauer PR, Burguera B, Ikramuddin S, et al. Effect of laparoscopic Roux-en Y gastric bypass on type 2 diabetes mellitus. Ann Surg. 2003;238:467-84.

20. • Cohen RV, Pinheiro JC, Schiavon CA et al. Effects of gastric bypass surgery in patients with type 2 diabetes and only mild obesity. Diabetes Care 2012;35:1420-8. First long term prospective follow up of metabolic surgery (RYGB) in patients with $B M I<35 \mathrm{~kg} / \mathrm{m} 2$.

21. Gill RS, Birch DW, Shi X, Sharma AM, Karmali S. Sleeve gastrectomy and type 2 diabetes mellitus: a systematic review. Surg Obes Relat Dis. 2011;6:707-13.

22. Dixon JB, O'Brien PE, Playfair J, et al. Adjustable gastric banding and conventional therapy for type 2 diabetes: a randomized controlled trial. JAMA. 2008;299:316-23.

23. Cohen RV, Schiavon CA, Pinheiro JS, Correa JL, Rubino F. Duodenal-jejunal bypass for the treatment of type 2 diabetes in patients with body mass index of $22-34 \mathrm{~kg} / \mathrm{m} 2$ : a report of 2 cases. Surg Obes Relat Dis. 2007;3:195-7.

24. Cohen R, Caravatto PP, Correa JL, et al. Glycemic control after stomach-sparing duodenal-jejunal bypass surgery in diabetic patients with low body mass index. Surg Obes Relat Dis. 2012;8:375-80. 
25. Escalona A, Yáñez R, Pimentel F, et al. Initial human experience with restrictive duodenal-jejunal bypass liner for treatment of morbid obesity. Surg Obes Relat Dis. 2010;6:126-31.

26. - Escalona A, Pimentel F, Sharp A et al. Weight loss and metabolic improvement in morbidly obese subjects implanted for 1 year with an endoscopic duodenal-jejunal bypass liner. Ann Surg. 2012;255:1080-5.

27. - Cohen RV, Neto MG, Correa JL et al. A pilot study of the duodenal-jejunal bypass liner in low body mass index type 2 diabetes. J Clin Endocrinol Metab. 2013;98:e279-82. First 2 reports of a non surgical metabolic intervention (endoscopic duodenal liner) and its outcomes over T2DM in mordly obese and overweight/obese population.

28. - Kashyap SR, Bhatt DL, Wolski K et al. Metabolic effects of bariatric surgery in patients with moderate obesity and type 2 diabetes: analysis of a randomized control trial comparing surgery with intensive medical treatment. Diabetes Care 2013. doi: 10.2337/dc12-1596. Two years follow up of Schauer's Stapede $R C T$ where it is reported the superiority of $R Y G B$ over $S G$ in metabolic control.

29. Strader AD. Ileal transposition provides insight into the effectiveness of gastric bypass surgery. Physiol Behav. 2006;88: 277-82.

30. - Stefater MA, Wilson-Perez HE, Chambers AP, Sandoval DA, Seeley RJ. All bariatric surgeries are not created equal: insights from mechanistic comparisons. Endocr Rev. 2012;33:595-622. Comprehensive review of mechanisms of action in metabolic surgery.

31. - Breen DM, Rasmussen BA, Kokorovic A et al. Jejunal nutrient sensing is required for duodenal-jejunal bypass surgery to rapidly lower glucose concentrations in uncontrolled diabetes. Nat Med. 2012;18:950-5.

32. - Jiao J, Bae EJ, Bandyopadhyay G et al. Restoration of euglycemia after duodenal bypass surgery is reliant on central and peripheral inputs in Zucker fa/fa rats. Diabetes 2013;62:1074-83.

33. - Salinari S, Debard C, Bertuzzi A et al. Jejunal proteins secreted by $\mathrm{db} / \mathrm{db}$ mice or insulin-resistant humans impair the insulin signaling and determine insulin resistance. PLoS One 2013;8:e56258. Three animal studies that reveal the important role of the duodenum in the pathophysiology of insulin resistance and back up the good outcomes that result from procedures that reroute the food through the gastrointestinal tract.
34. Wilson-Pérez HE, Chambers AP, Ryan KK, et al. Vertical sleeve gastrectomy is effective in two genetic mouse models of glucagon-like peptide-1 receptor deficiency. Diabetes. 2013; . doi: 10.2337/db12-1498.

35. - Liou AP, Paziuk M, Luevano J-M et al. Conserved shifts in the gut microbiota due to gastric bypass reduce host weight and adiposity. Sci Transl Med. 2013;5:178ra41. Elegant animal study of the role of micobiota changes in postoperative metabolic control and weight loss.

36. Yamaoka K, Tango T. Efficacy of lifestyle education to prevent type 2 diabetes: a meta-analysis of randomized controlled trials. Diabetes Care. 2005;28:2780-6.

37. Li Q, Chen L, Yang Z, et al. Metabolic effects of bariatric surgery in type 2 diabetic patients with lower body mass index. Diabetes Obes Metab. 2011;14:262-70.

38. •• Sjöström L, Peltonen M, Jacobson P et al. Bariatric surgery and long-term cardiovascular events. JAMA 2012;307:56-65. An arm of the SOS trial where is depicted that BMI is not correlated with cardiovascular events in diabetics.

39. Look AHEAD Research Group, Wing RR. Long-term effects of a lifestyle intervention on weight and cardiovascular risk factors in individuals with type 2 diabetes mellitus: four-year results of the Look AHEAD trial. Arch Intern Med. 2010;170:1566-75.

40. Gregg EW, Chen H, Wagenknecht LE, et al. Association of an intensive lifestyle intervention with remission of type 2 diabetes. JAMA. 2012;308:2489-96.

41. - Arterburn DE, Bogart A, Sherwood NE et al. A multisite study of long-term remission and relapse of type 2 diabetes mellitus following gastric bypass. Obes Surg. 2013;23:93-102. Good approach to factors involved in postoperative T2DM relapse.

42. - Arterburn DE, O'Connnor PJ. A look ahead at the future of diabetes prevention and treatment. JAMA 2012;308:2517-9. An Editorial on the importance of early intervention and further metabolic control and decreased cardiovascular events and deaths.

43. • Dixon JB, Zimmet P, Alberti KG, Rubino F. Bariatric surgery: an IDF statement for obese Type 2 diabetes. Diabet Med. 2011;28:628-42. The first step towards broadening surgical indications in uncontrolled T2DM.

44. Cohen R, Caravatto PP, Petry T. Metabolic surgery for type 2 diabetes in Patients with a BMI of $<35 \mathrm{~kg} / \mathrm{m}^{2}$ : a surgeon's perspective. Obes Surg. 2013. doi:10.1007/s11695-013-0930-2. 